\title{
A two-step quality-improvement intervention to address Pap smear quality at public health facilities in South Africa
}

\author{
M Mulongo, ${ }^{1} \mathrm{MB}$ BCh; A M Oberlin, ${ }^{1,2}$ C Firnhaber, ${ }^{1,3} \mathrm{MD}$, MS; B Goeieman, ${ }^{1} \mathrm{MB}$ BCh; S Ramotshela, ${ }^{1}$ P Michelow, ${ }^{4} \mathrm{MSc}, \mathrm{MB}$ BCh; \\ S Jordaan, ${ }^{4}$ C J Chibwesha, ${ }^{1,2} \mathrm{MD}, \mathrm{MSc}, \mathrm{FACOG}$ \\ ${ }^{1}$ Right to Care, Helen Joseph Hospital, Johannesburg, South Africa \\ ${ }^{2}$ Division of Global Women's Health, Department of Obstetrics and Gynecology, University of North Carolina at Chapel Hill, NC, USA \\ ${ }^{3}$ Division of Infectious Diseases, Department of Medicine, University of Colorado, Anschutz Outpatient Pavilion, Aurora, CO, USA \\ ${ }^{4}$ Department of Anatomical Pathology, Faculty of Health Sciences, University of the Witwatersrand, Johannesburg; and Cytology Unit, National \\ Health Laboratory Service, Johannesburg, South Africa
}

Corresponding author: C J Chibwesha (carla_chibwesha@med.unc.edu)

Background. The endocervical component of a Pap smear is an important indicator of sample quality - or 'adequacy'. However, only 6 of 52 districts in South Africa (SA) meet the Department of Health (DoH) performance benchmark: a 70\% adequacy rate. We implemented a quality-improvement (QI) intervention to address suboptimal Pap smear quality in Tshwane District, Gauteng Province, SA.

Objectives. To determine whether training with the wooden Ayre spatula (step 1) or introduction of the cytobroom (step 2) resulted in greater improvements in Pap smear adequacy rates.

Methods. Two Tshwane District health facilities participated in our QI project between May 2016 and February 2017. In step 1, staff received training on the Ayre spatula. In step 2, the spatula was replaced with the cytobroom. Pap smear volumes, adequacy rates and results are reported for the pre-intervention period and after each QI step. We compared adequacy rates using Fisher's exact test, with a significance level of $p=0.05$.

Results. In the pre-intervention period, 304 of 965 Pap smears were deemed adequate (32\%; 95\% confidence interval (CI) 29 - 35\%). After step 1, the proportion increased to 109 of 191 (57\%; 95\% CI $50-64 \%$; $p<0.01$ ). Similarly, after step 2, the proportion increased to 155 of 192 (81\%; 95\% CI $74-86 \% ; p<0.01)$. The proportion of abnormal smears increased from 13\% before the QI intervention to $17 \%$ after step 1 and $22 \%$ after step 2.

Conclusion. Although training in Pap smear collection using the Ayre spatula resulted in modest improvements in quality, facilities only achieved the DoH benchmark of a $70 \%$ adequacy rate after the introduction of the cytobroom.

S Afr Med J 2018;108(11):926-928. DOI:10.7196/SAMJ.2018.v108i11.13153

Cervical cancer is a leading cause of cancer death among women in low- and middle-income countries. ${ }^{[1]}$ In South Africa (SA), where the quality-improvement (QI) intervention was conducted, 1 in 32 women develop cervical cancer in their lifetime. ${ }^{[2]}$ Despite the existence of a national cervical screening programme, racial and socioeconomic inequities in cervical cancer incidence and death remain striking. ${ }^{[3,4]}$

When implemented effectively at scale, cervical screening programmes can substantially reduce the incidence of cervical cancer. ${ }^{[5-7]}$ However, screening coverage remains low throughout SA, with only 1 in 3 women receiving appropriate screening. ${ }^{\left[{ }^{[3}\right.}$ Furthermore, the quality of smears received by the National Health Laboratory Service (NHLS) varies. For example, the median adequacy rate - a measure of whether there is sufficient endocervical tissue for analysis - is $47 \%$, and many 'inadequate' smears must be repeated, resulting in unnecessary cost and attrition from care. ${ }^{[8]}$ One explanation for the low adequacy rate may be the choice of sample collection device. Currently, the vast majority of Pap smears are obtained using a wooden Ayre spatula, which is associated with suboptimal sampling of the endocervix and a higher rate of inadequate smears compared with use of the cytobroom. ${ }^{[9,10]}$

We sought to improve the quality of Pap smear screening in the Tshwane Department of Health (DoH) facilities using two targeted QI activities. Specifically, our objective was to determine whether training with the Ayre spatula (step 1) or introduction of the cytobroom (step 2) resulted in greater improvements in Pap smear adequacy rates.

\section{Methods}

Our QI project was conducted in collaboration with the Tshwane DoH. Located in northern Gauteng, there are $~ 90$ public health facilities that perform cervical screening in Tshwane District. We selected four candidate facilities, each performing at least 400 Pap smears per year, but with adequacy rates of $<40 \%$. Of these, two facilities located in urban Tshwane elected to participate.

In the pre-intervention period, between 2013 and 2014, we obtained aggregate-level data on the volume, adequacy rate and result of Pap smears performed at participating facilities. The two-step QI intervention was conducted between May 2016 and February 2017. Step 1 included 1 day of classroom training using the Ayre spatula, followed by 2 days of on-site mentorship. Step 2 included a 1-day on-site training in the use of the cytobroom. No additional mentorship was provided during step 2. De-identified Pap smear data were obtained from the NHLS for 2 - 3 months after each step of the intervention. Pap smears were reported by qualified cytotechnologists using the Bethesda system. ${ }^{[1]}$ All smears were processed and read in accordance with routine laboratory procedures, and the laboratory staff were unaware of the intervention. 
In this article, we describe Pap smear volumes and adequacy rates (proportion of samples with a representative endocervical component) for the pre-intervention period, as well as after each QI step. We also report the proportion, including 95\% confidence intervals (CIs), of abnormal Pap smears, defined as atypical squamous cells of undetermined significance or worse (ASCUS+), as this is the colposcopy referral threshold recommended by the $\mathrm{DoH}$ and World Health Organization (WHO). ${ }^{[3,12]}$ Hypothesis testing was performed using Fisher's exact tests, at a significance level of $p=0.05$.

\section{Ethical approval}

Ethical approval was granted by the University of the Witwatersrand Human Research Ethics Committee (ref. no. M150971) and the Tshwane Research Committee (ref. no. 07/2016).

\section{Results}

In the pre-intervention period (2013 - 2014), results were reported for 960 of 965 Pap smears performed at participating facilities. After step 1, which consisted of training and mentorship on the Ayre spatula, results were reported for 204 of 210 Pap smears. After step 2, during which the cytobroom was introduced, results were reported for 212 of 219 Pap smears. Because of laboratory error, some results were missing. The Pap smear adequacy rate during the pre-intervention period was 32\% (95\% CI 29 - 35\%) (Fig. 1). After step 1 , the adequacy rate increased to $57 \%$ (95\% CI 50 - 64\%; $p<0.01$ ). Similarly, after step 2, we observed further improvement in the Pap smear adequacy rate to $81 \%$ (95\% CI $74-86 \%$; $p<0.01)$. Results were similar when the analysis was performed disaggregated by clinic (data not shown).

With improvements in Pap smear adequacy rates throughout the QI project, we also observed increases in Pap smear abnormalities (Table 1). In the pre-intervention period, the proportion of abnormal results (ASCUS+) was 13\% (95\% CI $11-15 \%)$. After step 1, these rose to $17 \%$ ( $95 \%$ CI $12-23 \%$ ), while after step 2, 22\% were read as ASCUS+ (95\% CI 17 - 28\%).

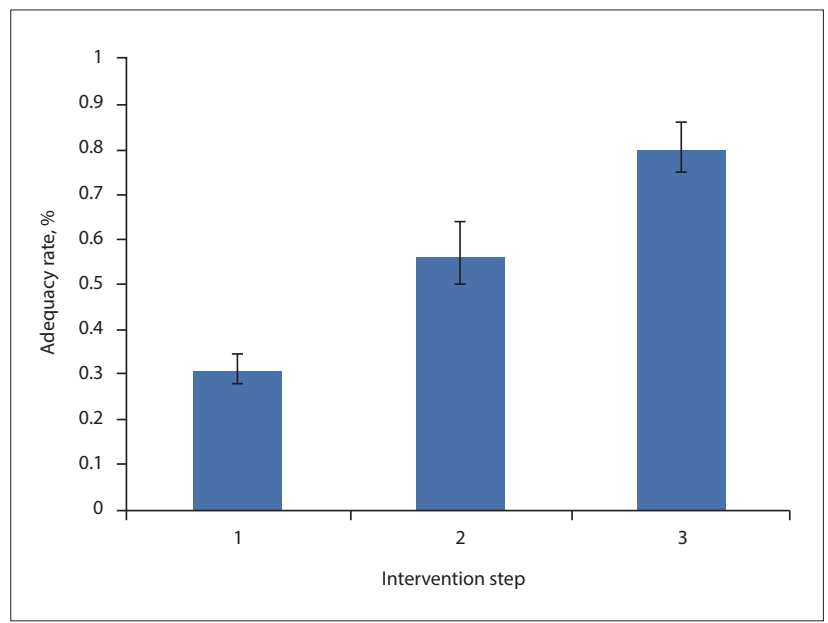

Fig. 1. Pap smear adequacy rates pre-intervention and after each step of the quality-improvement intervention.

\section{Discussion}

Although Pap smear quality is not determined solely by the presence of an endocervical component, we focused our QI project on Pap smear adequacy, as many public health facilities in SA consistently fall below the DoH benchmark for this metric. Our findings illustrate how simple QI interventions can improve Pap smear adequacy rates in public-sector health facilities. We found that both training on the Ayre spatula and introduction of the cytobroom resulted in statistically significant improvements in adequacy rates. We observed a $25 \%$ increase (from $32 \%$ to $57 \%$ ) in Pap smear adequacy after training and a further $24 \%$ rise after the introduction of the cytobroom (from $57 \%$ to $81 \%$ ).

We are not aware of any prior studies comparing training with the introduction of the cytobroom to improve Pap smear adequacy rates in SA. Our findings are consistent with those in the existing literature on Pap smear collection devices, demonstrating higher adequacy rates when Pap smears are performed using a cytobroom compared with a wooden spatula (odds ratio 1.6; 95\% CI 1.4 $1.7 \%){ }^{[9,10]} \mathrm{A}$ recent cost-effectiveness analysis indicated that using the cytobroom for Pap smear collection would save SA's public health system USD0.67 million per year. ${ }^{[13]}$ The modelling analysis also estimated that introducing the cytobroom would result in a $28 \%$ reduction in the number of women receiving false-negative diagnoses owing to increased identification of high-grade intraepithelial lesions. ${ }^{[13]}$

\section{Study limitations}

We noted several limitations of the project: (i) the number of participating clinics was small and both clinics were located in urban Gauteng, limiting the generalisability of our findings; (ii) we did not collect detailed clinic- or patient-level data and were unable to assess selection bias or examine other factors that may have influenced our findings; (iii) we did not consider other aspects of smear quality or address broader issues of access or coverage of cervical screening; and (iv) there may have been some variability in interpretation by different cytotechnologists. While these points may have introduced random error, they were not anticipated to have led to bias in our results in any systematic way. Future evaluations should be conducted with a larger, more representative sample of clinics and should assess patient-level factors on Pap smear adequacy rates.

\section{Conclusion}

Training and mentorship in the use of the Ayre spatula can significantly improve Pap smear adequacy rates. However, use of the cytobroom for Pap smear collection is the most efficient way to improve sample adequacy and DoH clinics should consider introducing the cytobroom as the standard collection device for Pap smears.

\section{Declaration. None.}

Acknowledgements. The authors acknowledge the Tshwane District Department of Health and the National Health Laboratory Service for their participation.

Table 1. Pap smear results before intervention and after each step of the quality-improvement intervention

\begin{tabular}{|c|c|c|c|c|c|c|}
\hline & \multicolumn{2}{|c|}{ Pre-intervention } & \multicolumn{2}{|c|}{ Post step 1} & \multicolumn{2}{|c|}{ Post step 2} \\
\hline & $n$ & $\%(95 \% \mathrm{CI})$ & $n$ & $\%(95 \% \mathrm{CI})$ & $n$ & $\%(95 \% \mathrm{CI})$ \\
\hline NILM & 838 & $87(85-89)$ & 170 & $83(77-88)$ & 165 & $78(72-83)$ \\
\hline ASCUS+ & 122 & $13(11-15)$ & 34 & $17(12-23)$ & 47 & $22(17-28)$ \\
\hline Total & 960 & 100 & 204 & 100 & 212 & 100 \\
\hline
\end{tabular}


Author contributions. CJC, $\mathrm{CF}$ and MM conceived and designed the project. MM, AMO, CF, BG, SR, PM, SJ and CJC participated in data collection and provided critical input with regard to the manuscript. AMO drafted and co-ordinated editorial comments regarding the manuscript. All authors approved the final version of the manuscript for publication.

Funding. Funding support for operational and quality improvement activities was provided by the US Agency for International Development (USAID) (grant no. 674-A-00-08-00007), the President's Emergency Plan for AIDS Relief (PEPFAR) (grant no. 674-A-12-00020) and the USAID Population, Health, and Environment (PHE) program (grant no. PHE ZA.09.0265). Trainee support (AMO) was provided by the UNC-Johns Hopkins-Morehouse-Tulane Fogarty Global Health Fellows Program (D43 TW009340).

\section{Conflicts of interest. None.}

1. Stewart BW, Wild CP. World Cancer Report 2014. Lyon, France: IARC Science Publications, 2014 2. Ervik M, Lam F, Ferlay J, Mery L, Soerjomataram I, Bray F. Cancer Today. Lyon, France: International Agency for Research on Cancer, 2016. http://gco.iarc.fr/today (accessed 5 December 2017).

3. National Department of Health. Cervical Cancer Prevention and Control Policy. Pretoria: NDoH, 2017.
4. National Institute for Communicable Diseases. South African National Cancer Registry. Cancer in South Africa. 2012. www.ncr.ac.za (accessed 10 January 2018)

5. Peirson L, Fitzpatrick-Lewis D, Ciliska D, Warren R. Screening for cervical cancer: A systematic review Peirson L, Fitzpatrick-Lewis D, Ciliska D, Warren R. Screening for cervical cances: As
and meta-analysis. Syst Rev 2013;2(35). https://doi.org/10.1186/2046-4053-2-35

Vicus D, Sutradhar R, Lu Y, Elit L, Kupets R, Paszat L. The association between cervical cancer Vicus D, Sutradhar R, Lu Y, Elit L, Kupets R, Paszat L. The association between cervical cancer
screening and mortality from cervical cancer: A population based case-control study. Gynecol Oncol screening and mortality from cervical cancer: A population base
2014;133(2):167-171. https://doi.org/10.1016/.ygyno.2014.02.037

7. Vaccarella S, Lortet-Tieulent J, Plummer M, Franceschi S, Bray F. Worldwide trends in cervical cance incidence: Impact of screening against changes in disease risk factors. Eur J Cancer 2013;49(15):32623273. https://doi.org/10.1016/j.ejca.2013.04.024

8. Makura C, Schnippel K, Michelow P, et al. Choropleth mapping of cervical cancer screening in South Africa using healthcare facility-level data from the national laboratory network. AIMS Public Health 2016;3(4):849-862. https://doi.org/10.3934/publichealth.2016.4.849

9. Martin-Hirsch P, Jarvis G, Kitchener H, Lilford R. Collection devices for obtaining cervical cytology samples. Cochrane Database Syst Rev 2000;(3):1-64. https://doi.org/10.1002/14651858.cd001036

10. O'Mahony D, Banach L, Igumbor E. A comparison of cervical smear adequacy using either the cytobrush or the Ayre spatula: A practice audit. S Afr Fam Pract 2006:48(9):15. https://doi.org/10.1 080/20786204.2006.10873457

11. Nayar R, Wilbur DC. The Bethesda System for Reporting Cervical Cytology: Definitions, Criteria, and Explanatory Notes. New York: Springer International Publishing, 2015.

12. World Health Organization. WHO Guidelines for Screening and Treatment of Precancerous Lesions 2. World Health Organization. WHO Guidelines for Scre
for Cervical Cancer Prevention. Geneva: WHO, 2013.

13. Schnippel K, Michelow P, Chibwesha CJ, et al. Cost-effectiveness of using the Cervex-Brush (broom) compared to the elongated spatula for collection of conventional cervical cytology samples within high-burden HIV setting: A model-based analysis. BMC Health Services Res 2015;15(1):499. https:// doi.org/10.1186/s12913-015-1163-y

Accepted 24 April 2018 